\title{
Successful Surgical Treatment of Descending Aorta Interruption: A Case Report
}

\author{
Pengxiong Zhu, MD, Xiaofeng Ye, MD, Qiang Zhao, MD
}

Department of Cardiac Surgery, Ruijin Hospital Shanghai Jiao Tong University School of Medicine, No.197, Ruijin Road (No.2), Shanghai, China

\section{ABSTRACT}

Descending aorta interruption is an extremely rare congenital defect. Conventional repair with end-to-end anastomoses is often a surgical challenge in view of the extensive collateral vessels that develop on the chest wall and inside the chest cavity. Extra-anatomic bypass is the preferred technique for the surgical repair of this entity, which avoids the network of collateral vessels, enables simultaneous treatment of associated lesions, and in all likelihood reduces morbidity and mortality. Here we describe an extra-anatomic bypass from the ascending aorta to the bilateral iliac arteries in a 24-year-old woman using vascular grafts (MAQUET Holding B.V. \& Co. KG, Rastatt, Germany) without cardiopulmonary bypass.

\section{INTRODUCTION}

Descending aorta interruption is a subtype of Type C interrupted aortic arch (IAA), depending on the site of discontinuity. The site of discontinuity may be distal to the left subclavian artery (Type A), between the left common carotid and the left subclavian arteries (Type B), or between the brachiocephalic and left common carotid arteries (Type C) [Tchervenkov 2005]. Given the low incidence of three per million live births and the high mortality of infants with untreated IAA - approximately $90 \%$ at the median age of four days- IAA is relatively seldom in adults, and only a few reports can be found in the world medical literature [Collins-Nakai 1976].

Various surgical techniques for IAA repair have been described, including anatomic and extra-anatomic bypass. The former technique has a high bleeding risk due to the extensive collateral vessels in the chest cavity [Matsui 2014]. Because of this, the latter technique-combining anastomoses for aorta reconstruction and repair of all coexisting intra-cardiac defects- is considered the optimal treatment. Described in this report is one case of descending aorta interruption, which was successfully treated with a bypass from the ascending aorta to the bilateral external iliac arteries.

Received November 13, 2016; received in revised form October 25, 2017; accepted April 13, 2018.

Correspondence: Dr. Qiang Zhao, Department of Cardiac Surgery, Ruijin Hospital Shanghai fiao Tong University School of Medicine, No.197, Ruijin Road (No.2), Shanghai, China 200025; +86-021-64370045 (e-mail: zq11607@rjh.com.cn).

\section{CASE REPORT}

A 24-year-old woman with a seven-year history of unexplained hypertension refractory to medical management was referred to our hospital with no obvious symptoms except

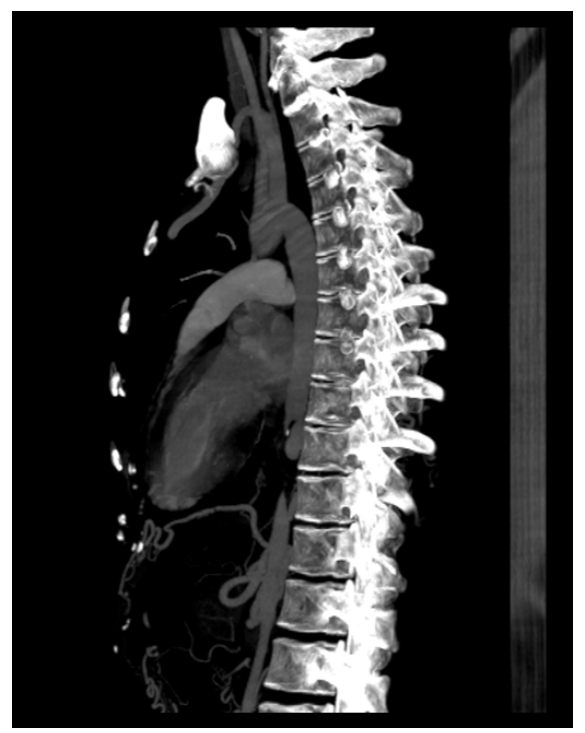

Figure 1. Chest and abdomen computed tomography showing a bird's beak appearance and total interruption of the descending aorta at level T9-T11, with tortuous and dilated collateral vessels.

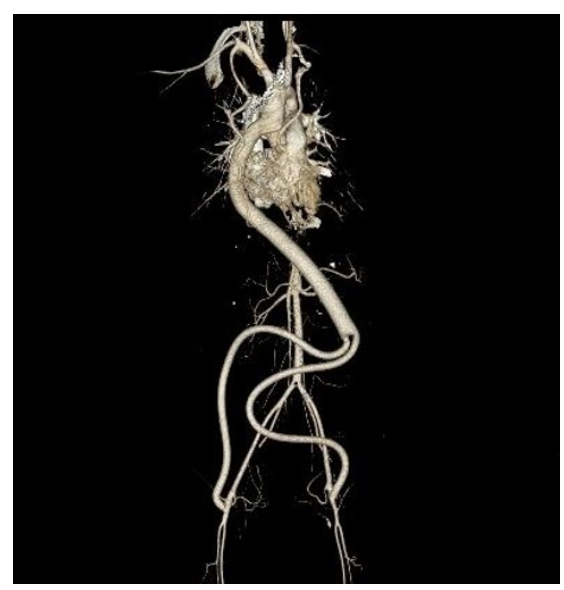

Figure 2. Postoperative computed tomography and three-dimensional reconstruction image demonstrating the patent graft. 
mild lower limb ache when sleeping, which could be reduced by peripatellapexy. She had a negative family history of hypertension, and no other risk factors (eg, obesity) for hypertension.

On examination, her heart rate was 100 beats/min with a regular rhythm and no heart murmurs. Her blood pressure was $140 / 70 \mathrm{mmHg}$ in the upper extremities, and 70/30 $\mathrm{mmHg}$ in the lower extremities, which meant her ankle-brachial index (ABI) was 0.5 , significantly lower than the normal range ( 0.9-1.3). Continuous murmurs around the abdominal aorta area and reduced pulsation of arteriae dorsalis pedis could be detected. Her muscle strength was normal, at $5 / 5$ in upper and lower extremities. There was no limb dyskinesia, sensory disturbances, or pathological reflex detected.

The levels of serum creatinine, urine protein, glomerular filtration rate, serum potassium, aldosterone renin ratio, serum cortisol, urine cortisol, serum thyroid stimulating hormone, and serum thyroid hormone were all within the normal ranges, which excluded a few causes of secondary hypertension, such as primary renal disease, primary aldosteronism, Cushing's syndrome and hypothyroidism. No obvious abnormalities could be detected by electrocardiogram or echocardiogram. Thoracic aorta computed tomography angiography (CTA) showed that there was a bird's beak appearance and total interruption of the descending aorta $5.0 \mathrm{~cm}$ in length at level T9-T11, located $1.6 \mathrm{~cm}$ above celiac artery. The tortuous and dilated collateral vessels were also noted (Figure 1). The patient was diagnosed with descending aorta interruption and secondary hypertension.

The surgical strategy of extra-anatomic repair- bypass from ascending aorta to bilateral external iliac arteries- was performed. This prevented the thoraco-abdominal conjunct incision applied during the replacement of the thoracoabdominal aorta that is used in the anatomic-repair technique. It also prevented the possible obstruction of visceral arteries that is complicated by interventional dilatation and stenting. We made a median sternotomy and bilateral inguinal incision for exposure of the ascending aorta and bilateral external iliac arteries. The bilateral external iliac arteries were dissected and looped. After the distance from ascending aorta to external iliac arteries was measured, a bifurcated vascular graft $(1.6 \mathrm{~cm} / 0.8 \mathrm{~cm} / 0.8 \mathrm{~cm})$ (MAQUET Holding B.V. \& Co. KG, Rastatt, Germany) was selected and extended with a straight graft $(1.6 \mathrm{~cm})$ (MAQUET Holding B.V. \& Co. KG, Rastatt, Germany), leaving the end-to-end anastomosis of the grafts in the pericardial cavity. With the help of endoscopic graspers, the route of the composite graft went from the right lateral side of the right atrium and passed through the anterior diaphragm and peritoneal cavity to both inguinal regions.

The distal anastomoses to external iliac arteries were performed first, then the proximal anastomosis on the right lateral aspect of the ascending aorta with a side-biting clamp. Both the proximal and distal anastomoses were done with a running polypropylene suture in an end-to-side fashion. Postoperative blood pressure was $120 / 70 \mathrm{mmHg}$ in the upper extremities and $130 / 80 \mathrm{mmHg}$ in the lower extremities. Thoracic aorta CTA with three-dimensional reconstruction images demonstrated good graft patency (Figure 2). The patient was discharged home uneventfully.

\section{DISCUSSION}

This case originated from the unexplained hypertension refractory to medical management. A number of medical entities may lead to secondary hypertension, including primary renal cause, primary aldosteronism, pheochromocytoma, Cushing disease, renovascular hypertension, and coarctation of aorta. Given the normal endocrine indexes and renal function, and the obviously low ABI, coarctation or interruption of aorta should be considered, although most cases are diagnosed in childhood.

IAA is characterized by the high mortality in neonates without treatment, and survival into adulthood is rare and dependent on substantial collateral circulation. Aortic interruption in adults leads to elevated blood pressure proximal to the interruption, increased cardiac afterload, and ischemic limbs and abdominal organs distal to the interruption, as well as reversible symptoms like "vascular paraplegia." However, symptoms of ischemia and congestive heart failure are not obvious in young IAA patients. Thus, more attention should be paid to an abnormal ABI with unexplained hypertension refractory to medical management. A chest computed tomography (CT), echocardiogram, and cardiac catheterization are recommended in the diagnosis of IAA. Extensive imaging may be warranted in these patients, given the high incidence of other cardiac and vascular abnormalities.

The management of IAA in adults appears to be the same as in infants, performed by either surgical repair or percutaneous stenting. In view of the extensive collateral vessels on the chest wall and inside the chest cavity, conventional repair of aortic interruption by end-to-end anastomoses is often a surgical challenge associated with a high risk of postoperative morbidity and mortality. Extra-anatomic repairs can decrease the risk potential complications.

Among the extra-anatomic techniques, we prefer a bypass from the ascending aorta to bilateral external iliac arteries, rather than a bypass to the abdominal aorta, where we would need to extend the incision to an upper midline laparotomy. Meanwhile, when compared with an anastomosis to abdominal aorta, the anastomoses to the bilateral external iliac arteries through inguinal incisions can be completed in more superficial sites, decreasing the intraoperative difficulty and making the surgery less invasive. An added advantage is the inconspicuous site of bilateral inguinal incisions.

\section{REFERENCES}

Collins-Nakai RL, Dick M, Parisi-Buckley L, et al. 1976. Interrupted aortic arch in infancy. J Pediatr 88:959-62.

Matsui H, Adachi I, Uemura H, et al. 2014. Anatomy of coarctation, hypoplastic and interrupted aortic arch: relevance to interventional/surgical treatment. Expert Rev Cardiovasc Ther 5:871-80.

Tchervenkov CI, Jacobs JP, Sharma K, et al. 2005. Interrupted aortic arch: surgical decision making. Semin Thorac Cardiovasc Surg Pediatr Card Surg Annu 8:92-102. 